ISSN: 2386-3919 - e-ISSN: 2386-3927

DOI: https://doi.org/10.14201/et2018361129149

\title{
CAUSAS DEL FRACASO ESCOLAR: UN ANÁLISIS DESDE LA PERSPECTIVA DEL PROFESORADO Y DEL ALUMNADO
}

\section{Causes of school failure: An analysis from the perspective of teachers and students}

\author{
A. M. ${ }^{a}$ Antelm lanzat, A. J. Gil lópez, M. L. Cacheiro González y E. Pérez Navío
}

Recibido: 8.01.2018; Aceptado: 10.3.2018; Publicado: 30.06.2018

Ref. Bibl. A. M. ANTElM LANZAT, A. J. GIL LÓPEZ, M. L. CACHEIRO GONZÁLEZ y E. PÉREZ NAVÍO. Causas del fracaso escolar: un análisis desde la perspectiva del profesorado y del alumnado. Enseñanza \& Teaching, 36, 1-2018, 129-149.

RESUMEN: El problema del fracaso escolar es un tema de especial actualidad, debido en gran parte al elevado porcentaje de alumnado que no termina con éxito la enseñanza obligatoria. El fracaso escolar, al igual que el éxito escolar, está determinado por múltiples causas, componentes o factores. Así, se señalan causas relacionadas con el estudiante, la escuela y la familia. Este trabajo tiene como objetivo analizar las causas del fracaso escolar desde la perspectiva del alumnado, el profesorado y los equipos directivos de educación secundaria. Se lleva a cabo un estudio descriptivo con enfoque cuantitativo. La información se recoge a través de un cuestionario, con validez y fiabilidad contrastada, en sus distintas dimensiones. Los participantes de este estudio se corresponden con 757 alumnos, 221 profesores y 116 directivos y jefes de departamento de 17 Institutos de Enseñanza Secundaria de la provincia de Valencia. Los resultados indican que las causas más importantes de fracaso escolar son, en primer lugar, el compromiso del alumno con el estudio y, en segundo lugar, la implicación de la familia en la educación. Más especialmente el alumnado da importancia al esfuerzo, el profesorado a los problemas de socialización del alumnado y los equipos directivos a la responsabilidad del alumno y de la familia en el proceso de aprendizaje. Además, se comprueba que existen diferencias 
A. M. ANTELM, A. J. GIL, M. L. CACHEIRO Y E. PÉREZ NAVÍO

CAUSAS DEL FRACASO ESCOLAR: UN ANÁLISIS DESDE LA PERSPECTIVA DEL PROFESORADO Y DEL ALUMNADO

estadísticamente significativas en las respuestas del alumnado, el profesorado y los equipos directivos. Por último, se discuten los resultados.

Palabras clave: fracaso escolar; educación secundaria; ambiente escolar; sistema educativo; análisis cuantitativo.

SUMMARY: The problem of school failure is a topic of special relevance, due in large part to the high percentage of students who do not successfully complete compulsory education. School failure, as well as school success, is determined by multiple causes, components or factors, as well as causes related to the student, the school and the family. The objective of this work is to analyze the causes of school failure from the perception of students, teachers and secondary school management teams. A descriptive study with a quantitative approach is carried out. The information is collected through a questionnaire, with proven validity and reliability, in its different dimensions. The participants in the study are: 757 students, 221 professors and 116 managers and heads of department of 17 Educational Centers of Valencia. The results indicate that the most important causes of school failure are, first, the student's commitment to the study and, secondly, the involvement of the family in education. More especially the students give importance to the effort, the teachers to the socialization problems of the students and the management teams to the responsibility of the student and the family in the learning process. In addition, it is verified that there are statistically significant differences in the responses of the students, the professed and the management teams. Finally, the results are discussed.

Key words: school failure; secondary education; school environment; educational system; quantitative analysis.

\section{INTRODUCCIÓN}

Las altas tasas de abandono y fracaso escolar en el conjunto de la Unión Europea y específicamente en España han puesto de actualidad el problema del fracaso escolar. Como señalan Ritacco y Amores (2016), el estudio del fenómeno del fracaso escolar lleva consigo acercarse a la problemática realidad de la exclusión social y educativa. Los estudiantes que no terminan los estudios obligatorios, junto con otros factores, tienen un mayor riesgo de exclusión laboral (Melendro, 2008; Pérez, 2008) y social (Calero, Choi y Waisgrais, 2010). Asimismo, se ha señalado que el nivel educativo alcanzado por una persona es un determinante significativo de sus posibilidades de desarrollo personal y profesional (Levin, 2003). De manera indirecta, señalan Espínola y Claro (2010), el que una persona no concluya con éxito la educación secundaria tiene importantes implicaciones en los programas de empleo, en la capacitación laboral y en la productividad económica. Todo ello confirma las importantes repercusiones que genera el fracaso escolar, y que afectan a la persona, a la economía y a la sociedad en su conjunto. 
A. M. ANTELM, A. J. GIL, M. L. CACHEIRO Y E. PÉREZ NAVÍO DEL PROFESORADO Y DEL ALUMNADO

El fracaso escolar, al igual que el éxito escolar, está determinado por múltiples causas, componentes o factores, así, se habla de causas sociales, económicas y educativas (Roca, 2010). De Witte, Cabus, Thyssen, Groot y Brink (2014) clasifican los predictores de fracaso escolar más comunes en la literatura en factores del estudiante, familiares, de la escuela y de la comunidad. Lozano (2012) destaca tres tipos factores que intervienen en el fracaso escolar: el familiar, el académico y el personal (los propios del estudiante). Ritacco y Amores (2016) enfatizan que los estudiantes "fracasan no solo por dificultades de aprendizaje o por problemas personales relacionados con su entorno familiar, sino que también se estrellan contra un sistema educativo que no ha sido capaz de proporcionar las respuestas adecuadas a sus necesidades» (p. 138). Además, habría que considerar las mutuas relaciones e influencias entre estos factores o variables de éxito y fracaso escolar (González, Caso, Díaz y López, 2012), lo que vendría a señalar la dificultad de abordar este problema.

Por ello, un análisis del fracaso escolar desde diversas perspectivas puede ayudar a explicar con mayor profundidad algunas de sus posibles causas. Dicho de otro modo, un análisis a través de la información de distintos sujetos implicados en el problema, por ejemplo, el alumnado y el profesorado, permite comparar distintas perspectivas y aportar elementos de reflexión para posibles intervenciones en este problema. Por ejemplo, puede ayudar a implantar programas de retención académica (Tinto, 1992), pues la implementación de este tipo de programas surge de la autoevaluación de los miembros de la organización escolar, entre los que se encuentran los alumnos, los profesores y los equipos directivos (Aguado, Aguilar y González, 2009).

Este trabajo tiene como objetivo general analizar las causas del fracaso escolar. Para la investigación empírica se recoge información de tres grupos de sujetos implicados en el fracaso escolar: el alumnado próximo a terminar la Educación Secundaria Obligatoria (ESO), el profesorado en funciones de aula y el profesorado en funciones directivas de los Institutos de Enseñanza Secundaria (IES) en los que estudian estos alumnos. La información se recoge por medio de tres cuestionarios a tres tipos de personas: 757 estudiantes, 221 docentes y 116 directivos y jefes de departamento de 17 IEs de la provincia de Valencia, en España, a través de un muestreo no probabilístico.

\section{El PROBLEMA DEL FRACASO ESCOLAR}

En este punto se presenta el concepto y causas de fracaso escolar y se señalan algunos estudios que han analizado algunas posibles causas de fracaso escolar.

No existe unanimidad para definir el concepto de fracaso escolar. En el informe de la OCDE sobre fracaso escolar se indican tres manifestaciones de este fenómeno (Marchesi, 2003: 7):

La primera se refiere a los alumnos con bajo rendimiento académico, es decir aquellos que a lo largo de su escolarización no alcanzan un nivel de conocimientos 
A. M. ANTELM, A. J. GIL, M. L. CACHEIRO Y E. PÉREZ NAVÍO CAUSAS DEL FRACASO ESCOLAR: UN ANÁLISIS DESDE LA PERSPECTIVA DEL PROFESORADO Y DEL ALUMNADO

mínimo. La segunda abarca a los alumnos que abandonan o terminan la educación obligatoria sin la titulación correspondiente. La tercera apunta a las consecuencias sociales y laborales en la edad adulta de los alumnos que no han alcanzado la preparación adecuada.

En este trabajo se sigue la segunda de las manifestaciones y, en línea con otros autores, consideramos fracaso escolar la proporción de individuos que no consiguen concluir los estudios obligatorios (Calero et al., 2010). En España, se consideraría el fracaso escolar cuando el alumnado no ha terminado con éxito la Educación Secundaria Obligatoria (ESO) y, por ello, no recibe el título de la EsO.

El fracaso escolar es un concepto multifactorial y complejo (Perreanoud, 1990), pues depende de su medición y de los niveles en los cuales se centra el estudio (Martínez-Otero, 2009). En este sentido, la literatura empírica ha identificado numerosos determinantes o causas de fracaso escolar, entre los que se destacarían: el género, las características parentales o el nivel de educación de los padres (Bratti, 2007; Oreopoulos, 2007). En términos generales, se podría hablar de grupos de causas que determinarían el fracaso escolar.

El primer grupo de causas se relaciona con las características individuales de los estudiantes, como su nivel de motivación, el esfuerzo y la percepción a apoyo de los padres (Engin-Demir, 2009); estas causas estarían ligadas con los problemas de aprendizaje del alumno y con su falta de esfuerzo o implicación en el aprendizaje (Castejón y Vera, 1996).

El segundo grupo de causas analiza los factores sociales relacionados con las características de la familia del alumno, como sus posibilidades económicas o sus características culturales, entre otras causas, y se relaciona con la incapacidad de la sociedad para asegurar una respuesta productiva, cultural o igualadora del sistema educativo (Mena, Fernández y Riviére, 2010).

El tercer grupo de causas gira en torno al funcionamiento del sistema educativo, y se refiere, entre otros aspectos, a la gestión de los centros educativos y la práctica docente (Escudero y Martínez, 2012). Esta última perspectiva se relaciona, entonces, con los agentes más directamente implicados en el sistema educativo: la administración educativa y el profesorado. En el ámbito de la administración se estudian temas relacionados con la legislación, con el sistema escolar y con la organización de centros. Por ejemplo, Willms y Somers (2001) mostraron un efecto positivo significativo en los resultados escolares y la proporción discente-docente en el aula. En relación al profesorado se analiza, por ejemplo, su preparación y su estilo de enseñanza.

Diversos trabajos han estudiado la incidencia de varios grupos de causas en el fracaso escolar. La investigación de Antelm, Gil y Cacheiro (2015), llevada a cabo con alumnos de la ESO, señala 4 grupos de causas de fracaso escolar, el primer grupo, la intervención del alumno y la familia en el aprendizaje; el segundo grupo, la socialización del alumno en la escuela y la familia; el tercer grupo, la capacitación del alumno; y el cuarto grupo, la situación económica de la familia del 
A. M. ANTELM, A. J. GIL, M. L. CACHEIRO Y E. PÉREZ NAVÍO DEL PROFESORADO Y DEL ALUMNADO

alumno. Las causas más destacadas de fracaso escolar resultan las del primer grupo, las relacionadas con las características del alumno y de su familia.

En el trabajo de Hafiane, Kaddari, Elachqar, Elaissaoui y Tahraoui (2015) se pone de manifiesto que el género tiene un efecto significativo en los niveles de fracaso escolar, siendo las chicas las que tienen menos tasa de fracaso escolar. Entre las variables sobre antecedentes familiares se encontró que el nivel de educación de los padres (especialmente el nivel educativo de las madres) era significativo en los problemas de fracaso escolar. Por el contrario, el tamaño del hogar no tuvo un efecto significativo en la tasa de fracaso escolar, lo que vendría a señalar que la variación en el número de hermanos no afecta a la atención que los padres prestan a sus hijos.

Lozano (2012) encuestó a 1.178 estudiantes de los 4 cursos de enseñanza secundaria de Andalucía para analizar el fracaso escolar a través de un cuestionario cuyas dimensiones eran: motivaciones, afectivas y sociofamiliares en el entorno académico. Los resultados muestran una influencia directa del nivel académico de los padres, el género, la motivación y las relaciones entre compañeros en el fracaso escolar.

En las conclusiones del estudio de Calero et al. (2010) se destaca que dentro de las variables de ámbito personal el género incide en el fracaso escolar, teniendo las chicas menor probabilidad de fracaso. Las variables relacionadas con el ámbito familiar determinan, también, un alto grado de riesgo en el fracaso escolar, siendo aquellos hogares que están mejor dotados de recursos materiales educativos, y hacen mejor uso de los mismos, los que tienen una menor probabilidad de fracaso escolar. Por último, se destaca que las políticas destinadas a la agrupación de alumnos, entre y dentro de las clases, tienen un nulo impacto para reducir el riesgo de fracaso escolar en el centro.

En la investigación de Fernández y Rodríguez (2008) se plantean 4 factores del fracaso escolar: la clase social de origen del estudiante, la profesión de sus padres, la estructura de su familia y, finalmente, el género del estudiante. De este trabajo destacan algunas conclusiones: los chicos de clases bajas tienen más probabilidades de haber repetido curso, la probabilidad de repetir curso es mayor entre estudiantes que viven en hogares monoparentales, no se encuentra evidencia de que los estudiantes inmigrantes o hijos de inmigrantes o los hijos de una madre laboralmente activa repitan más. Y, por último, las chicas tienden a repetir curso sustancialmente menos que los chicos.

El fracaso escolar es un fenómeno que no responde a una única causa, sino a un conjunto de factores que interactúan y que contribuyen a que un estudiante no concluya con éxito la enseñanza obligatoria. Este grupo de causas se vinculan con aspectos relacionados con las actitudes de los alumnos hacia la enseñanza, su autoeficacia (Britner y Pajares, 2006) o sus intereses (Swarat, Ortony y Revelle, 2012) y, también, con la relación del alumno con su entorno más cercano (Cabo y Romero, 2014) y con la gestión del sistema educativo (Willms y Somers, 2001).

Como causas de fracaso escolar se han señalado los comportamientos antisociales que llevan a la expulsión del alumnado del centro escolar. En el estudio 
A. M. ANTELM, A. J. GIL, M. L. CACHEIRO Y E. PÉREZ NAVÍO CAUSAS DEL FRACASO ESCOLAR: UN ANÁLISIS DESDE LA PERSPECTIVA DEL PROFESORADO Y DEL ALUMNADO

de Robison, Jaggers, Rhodes, Blackmon y Church (2017) se comprobó que ser expulsado de las aulas le da al estudiante un promedio de un $8 \%$ de probabilidad de abandonar la escuela y un $28 \%$ de posibilidades de graduarse a tiempo, frente al $4 \%$ y un $57 \%$, respectivamente para aquellos alumnos que no son expulsados.

También se han estudiado los efectos de los rasgos de personalidad en el fracaso escolar, en especial sus combinaciones. En la investigación de Migali y Zucchelli (2017) se encontró que la introversión y un cierto rasgo de sujeto identificado como neurasténico se asocian individualmente con mayores posibilidades de abandono escolar. Asimismo, se encontró que los individuos que renuncian al cuidado de la salud tienen un mayor riesgo de fracaso escolar.

Otros trabajos se han acercado teóricamente al problema del fracaso escolar. González (2006) señala cuatro aspectos de fracaso o abandono escolar. El primero, el clima escolar, la ausencia de lazos sociales entre alumno y profesor es una causa importante de abandono escolar. El segundo, las políticas o los procedimientos respecto a la asistencia a clase, las políticas de centro en el que se penalizan las ausencias tienen un efecto positivo en el incremento de la asistencia, pero una política de "tolerancia cero" es perjudicial. El tercero, las estructuras organizativas, las estructuras departamentalizadas propias de los IES, por ejemplo, el hecho de que el profesor cambie de grupo de sesión de clase conduce a que el profesorado tenga una orientación hacia la asignatura y no hacia el alumno. El cuarto, el currículo escolar, un currículo que proporcione experiencias escolares motivadoras que atienda al desarrollo intelectual de los alumnos contribuye a prevenir el abandono escolar.

\section{MÉTODO}

\subsection{Objetivos}

Los objetivos de la investigación son los siguientes:

- Conocer la percepción sobre posibles causas de fracaso escolar desde la perspectiva del alumnado de cuarto curso de la ESO, del profesorado en funciones de aula y del profesorado en funciones directivas (equipos directivos y jefes de departamento).

- Examinar la relación entre los distintos tipos de causas según la perspectiva del alumnado de cuarto curso de la ESO, del profesorado en funciones de aula y del profesorado en funciones directivas (equipos directivos y jefes de departamento).

- Comparar las respuestas sobre percepción de posibles causas de fracaso escolar desde la perspectiva del alumnado de cuarto curso de la ESO, del profesorado en funciones de aula y del profesorado en funciones directivas (equipos directivos y jefes de departamento). 


\subsection{Hipótesis}

Para comparar las respuestas entre los sujetos que intervienen en el estudio, se proponen las siguientes hipótesis (Tabla 1).

\section{TABLA 1}

Hipótesis en relación con las variables del fracaso escolar

\begin{tabular}{|c|l|}
\hline HIPÓTESIS & \multicolumn{1}{c|}{ DESCRIPCIÓN DE LA HIPÓTESIS } \\
\hline H1 & $\begin{array}{l}\text { No existen diferencias significativas entre las respuestas del alumnado, del } \\
\text { profesorado y de los equipos directivos sobre las causas de fracaso escolar. }\end{array}$ \\
\hline H2 & $\begin{array}{l}\text { No existen diferencias significativas entre las respuestas del alumnado, y del } \\
\text { conjunto de profesorado (profesores y equipos directivos) sobre las causas } \\
\text { de fracaso escolar. }\end{array}$ \\
\hline H3 & $\begin{array}{l}\text { No existen diferencias significativas entre las respuestas del profesorado y } \\
\text { de los equipos directivos sobre las causas de fracaso escolar. }\end{array}$ \\
\hline
\end{tabular}

\subsection{Participantes}

El trabajo se ha llevado a cabo en 3 grupos de sujetos en 17 IES de enseñanza: (1) el alumnado del último curso de la ESO; (2) el profesorado de este nivel educativo; y (3) los equipos directivos de estos IES.

Los alumnos participantes han sido de 757 sujetos, de los cuales el 48,7\% es hombre y el 51,3\% es mujer. Su media de edad es de 15,75 años con una desviación típica de 0,83 .

El profesorado participante ha sido de 221 sujetos, de los cuales el 46,6\% es hombre y el $53,4 \%$ es mujer. Su media de edad es de 46,87 años con una desviación típica de 7,98.

Los directivos que han participado en el estudio han sido 116 sujetos, de los cuales el $43,1 \%$ es hombre y el $56,9 \%$ es mujer. Su media de edad es de 56,9 años con una desviación típica de 7,73.

Los 17 IEs pertenecientes a la provincia de Valencia se han seleccionado a través de un muestreo aleatorio por conveniencia según la ubicación de las comarcas en las que se divide la provincia, se ha recogido información en 6 de ellas: Horta Nord (5 IES), Ciudad de Valencia (2 IES), Poblats Maritims (3 IES), Horta Sud (3 IES), Camp Turia (3 IES), y Alto Palancia (1 IES).

Los IES representan la variedad de centros educativos situados en distintas comarcas, con distintas condiciones socioeconómicas, siendo la mayoría de titularidad pública. 
A. M. ANTELM, A. J. GIL, M. L. CACHEIRO Y E. PÉREZ NAVÍO CAUSAS DEL FRACASO ESCOLAR: UN ANÁLISIS DESDE LA PERSPECTIVA DEL PROFESORADO Y DEL ALUMNADO

\subsection{Instrumento}

Para la recogida de información se ha utilizado el cuestionario de Antelm et al. (2015) que investiga las causas del fracaso escolar en una muestra de alumnado de ESO en España. El cuestionario consta de 16 ítems, que abordan las causas de fracaso escolar relacionadas con el alumnado y el contexto escolar y familiar del alumno. El cuestionario consta de 4 escalas y una escala Likert que de 1 a 7 desde un menor hasta un mayor acuerdo:

(1) Primera escala: La intervención del alumno y la familia en el aprendizaje (6 ítems).

(2) Segunda escala: La socialización del alumno en el contexto escolar y familiar ( 5 ítems).

(3) Tercera escala: La capacitación del alumno (3 ítems).

(4) Cuarta escala: La situación económica de la familia del alumno (2 ítems).

En la Tabla 2 se presentan los análisis de fiabilidad a través del alpha de Cronbach, para cada una de las escalas del cuestionario.

TABLA 2

Análisis de fiabilidad de las escalas para los tres tipos de informantes: alumnado, profesores y directivos

\begin{tabular}{|l|c|c|c|}
\hline \multicolumn{1}{|c|}{ EsCALAS } & ALUMNADO & PROFESORES & DireCTIVOS \\
\hline & \multicolumn{2}{|c|}{ "ALPHA DE CRONBACH» } \\
\hline $\begin{array}{l}\text { La intervención del alumno y la familia en el } \\
\text { aprendizaje }\end{array}$ & 0,865 & 0,665 & 0,786 \\
\hline $\begin{array}{l}\text { La socialización del alumno en la escuela y } \\
\text { la familia }\end{array}$ & 0,794 & 0,764 & 0,787 \\
\hline La capacitación del alumno & 0,650 & 0,748 & 0,605 \\
\hline $\begin{array}{l}\text { La situación económica de la familia del } \\
\text { alumno }\end{array}$ & 0,679 & 0,634 & 0,700 \\
\hline
\end{tabular}

Como se observa en la Tabla 2, para cada una de las escalas y, en todos los casos, el alpha de Cronbach es superior a 0,600 por lo que se consideran aceptables (Rindskopf, 2015), especialmente en etapas de investigación temprana como es nuestro caso, no obstante, en posteriores investigaciones se trataría de aumentar el número de ítems en cada una de las escalas que contribuyera a elevar su fiabilidad. Además, se calculó el coeficiente alpha de Cronbach para el total de los ítems del cuestionario para los tres tipos de informantes. Los alpha de Cronbach resultaron: 0,881 para el cuestionario del alumnado; 0,836 para el profesorado; y 0,841 para el cuestionario de los directivos. Siendo en todos los casos superior al 0,70 (Nunnally y Berstein, 1994), evidenciando su fiabilidad. 


\subsection{Procedimiento y tipo de estudios}

Para llevar a cabo el estudio se recabó la autorización de la Consejería de Educación de la Generalitat de Valencia para la recogida de los cuestionarios al alumnado, profesorado y directivos de los 17 IES. La entrega de cuestionarios se llevó a cabo por el propio equipo de investigación, que más tarde los recogió una vez que habían sido cumplimentados. Los cuestionarios fueron administrados entre los meses de abril, mayo y junio de 2015.

Se realiza un estudio cuantitativo a través de encuesta a alumnos, profesores y directivos sobre posibles causas de fracaso escolar. Se llevan a cabo 3 tipos de estudios: (1) análisis univariado, para conocer los resultados descriptivos del estudio; (2) análisis de correlación, que nos permite conocer las relaciones entre las variables propuestas; y (3) análisis de varianza, para contrastar las hipótesis.

\section{ANÁLISIS E INTERPRETACIÓN DE RESULTADOS}

En primer lugar, se presentan los resultados del análisis descriptivo, que permite conocer las valoraciones de los 3 grupos de informantes -alumnado, profesorado y equipo directivo- de cada una de las escalas y de los ítems del cuestionario. En segundo lugar, se realiza un análisis de correlación de Pearson, para conocer la covariación entre las escalas del cuestionario. Y, en tercer lugar, se lleva a cabo un análisis de varianza, ANOVA de un factor, para comparar las respuestas entre los grupos de informantes.

\subsection{Resultados del estudio descriptivo}

La Tabla 3 presenta las puntuaciones medias y las desviaciones típicas de los resultados de los tres grupos de informantes, en su conjunto y cada uno de ellos por separado.

\section{TABLA 3}

Puntuaciones medias de escalas e ítems del conjunto de informantes y de cada uno de ellos - alumnado, profesorado y equipos directivos (escala de 1 a 7)

\begin{tabular}{|c|c|c|c|c|}
\hline \multicolumn{1}{|c|}{ Escalas E Ítems } & TOTAL & Alumnado & Profesorado & Directivos \\
\hline & \multicolumn{3}{|c|}{ MEDIAS (DESVIACIONES TíPICAS) } \\
\hline $\begin{array}{l}\text { La intervención del alumno y la } \\
\text { familia en el aprendizaje }\end{array}$ & $\begin{array}{c}5,08 \\
(1,30)\end{array}$ & $4,78(1,39)$ & $5,80(0,65)$ & $5,64(0,82)$ \\
\hline $\begin{array}{l}\text { 1. El compromiso del alumno } \\
\text { con el estudio }\end{array}$ & $\begin{array}{c}5,36 \\
(1,78)\end{array}$ & $5,01(1,90)$ & $6,29(0,99)$ & $5,91(1,33)$ \\
\hline $\begin{array}{l}\text { 2. El apoyo familiar hacia el } \\
\text { alumno }\end{array}$ & $\begin{array}{c}5,18 \\
(1,83)\end{array}$ & $4,79(1,98)$ & $6,10(0,93)$ & $5,98(1,14)$ \\
\hline
\end{tabular}


A. M. ANTELM, A. J. GIL, M. L. CACHEIRO Y E. PÉREZ NAVÍO CAUSAS DEL FRACASO ESCOLAR: UN ANÁLISIS DESDE LA PERSPECTIVA DEL PROFESORADO Y DEL ALUMNADO

\begin{tabular}{|c|c|c|c|c|}
\hline ESCALAS E ÍTEMS & TOTAL & ALUMNADO & PROFESORADO & DiRECTIVOS \\
\hline & \multicolumn{4}{|c|}{ MEDIAS (DESVIACIONES TÍPICAS) } \\
\hline $\begin{array}{l}\text { 3. Las expectativas del alumno } \\
\text { hacia el curso }\end{array}$ & $\begin{array}{c}4,92 \\
(1,61)\end{array}$ & $4,81(1,75)$ & $5,19(1,27)$ & $5,09(1,16)$ \\
\hline $\begin{array}{l}\text { 4. La responsabilidad del alumno } \\
\text { hacia el trabajo diario }\end{array}$ & $\begin{array}{c}5,39 \\
(1,59)\end{array}$ & $5,06(1,70)$ & $6,20(0,85)$ & $6,03(1,09)$ \\
\hline $\begin{array}{l}\text { 5. La percepción de Autoeficacia } \\
\text { del alumno }\end{array}$ & $\begin{array}{c}4,60 \\
(1,46)\end{array}$ & $4,51(1,50)$ & $4,83(1,35)$ & $4,72(1,32)$ \\
\hline $\begin{array}{l}\text { 6. La implicación de la familia en } \\
\text { la educación }\end{array}$ & $\begin{array}{c}5,03 \\
(1,85)\end{array}$ & $4,51(1,91)$ & $6,21(0,92)$ & $6,11(1,06)$ \\
\hline $\begin{array}{l}\text { La socialización del alumno en la } \\
\text { escuela y la familia }\end{array}$ & $\begin{array}{c}4,61 \\
(1,28)\end{array}$ & $4,31(1,32)$ & $5,28(0,87)$ & $5,31(0,87)$ \\
\hline $\begin{array}{l}\text { 7. La falta de integración del } \\
\text { alumno en el aula }\end{array}$ & $\begin{array}{c}4,66 \\
(1,87)\end{array}$ & $4,25(1,95)$ & $5,62(1,19)$ & $5,43(1,39)$ \\
\hline $\begin{array}{l}\text { 8. La existencia de situaciones } \\
\text { conflictivas en la familia }\end{array}$ & $\begin{array}{c}5,00 \\
(1,78)\end{array}$ & $4,56(1,88)$ & $5,92(1,06)$ & $6,07(0,92)$ \\
\hline $\begin{array}{l}\text { 9. La falta de habilidad del } \\
\text { alumno para pedir ayuda a los } \\
\text { profesores }\end{array}$ & $\begin{array}{c}4,70 \\
(1,61)\end{array}$ & $3,39(1,69)$ & $5,46(1,15)$ & $5,24(1,13)$ \\
\hline $\begin{array}{l}\text { 10. La resistencia a la frustración } \\
\text { del alumno o la alumna }\end{array}$ & $\begin{array}{c}4,58 \\
(1,57)\end{array}$ & $4,41(1,64)$ & $4,85(1,40)$ & $5,17(1,17)$ \\
\hline $\begin{array}{l}\text { 11. La habilidad del alumno } \\
\text { para pedir ayuda a sus } \\
\text { compañeros }\end{array}$ & $\begin{array}{c}4,16 \\
(1,64)\end{array}$ & $3,95(1,75)$ & $4,59(1,27)$ & $4,65(1,22)$ \\
\hline La capacitación del alumno & $\begin{array}{c}4,50 \\
(1,25)\end{array}$ & $4,24(1,28)$ & $5,09(1,05)$ & $5,01(0,85)$ \\
\hline $\begin{array}{l}\text { 12. El cociente intelectual del } \\
\text { alumno o la alumna }\end{array}$ & $\begin{array}{c}4,16 \\
(1,66)\end{array}$ & $3,94(1,77)$ & $4,71(1,28)$ & $4,58(1,22)$ \\
\hline $\begin{array}{l}\text { 13. Las dificultades de } \\
\text { aprendizaje del alumno }\end{array}$ & $\begin{array}{c}4,90 \\
(1,61)\end{array}$ & $4,61(1,69)$ & $5,60(1,22)$ & $5,51(1,05)$ \\
\hline $\begin{array}{l}\text { 14. La capacidad de } \\
\text { autoevaluación del alumno }\end{array}$ & $\begin{array}{c}4,44 \\
(1,50) \\
\end{array}$ & $4,20(1,53)$ & $4,98(1,35)$ & $4,96(1,15)$ \\
\hline $\begin{array}{l}\text { La situación económica de la } \\
\text { familia del alumno }\end{array}$ & $\begin{array}{c}4,16 \\
(1,39)\end{array}$ & $4,03(1,45)$ & $4,32(1,23)$ & $4,70(1,04)$ \\
\hline $\begin{array}{l}\text { 15. Los recursos económicos de } \\
\text { la familia del alumno }\end{array}$ & $\begin{array}{c}4,14 \\
(1,58)\end{array}$ & $4,01(1,64)$ & $4,30(1,45)$ & $4,71(1,25)$ \\
\hline $\begin{array}{l}\text { 16. La situación laboral de la } \\
\text { familia }\end{array}$ & $\begin{array}{c}4,19 \\
(1,60)\end{array}$ & $4,05(1,69)$ & $4,35(1,42)$ & $4,77(1,13)$ \\
\hline
\end{tabular}


A. M. ANTELM, A. J. GIL, M. L. CACHEIRO Y E. PÉREZ NAVÍO DEL PROFESORADO Y DEL ALUMNADO

Como muestra la Tabla 3, se podrían destacar dos resultados generales, el primero, que la gran mayoría de las causas de fracaso escolar recibe una alta puntuación, por encima de la media en la escala Likert de 1 a 7 puntos y, el segundo, que existe semejanza en las valoraciones por los 3 colectivos de informantes, con algunas excepciones, por ejemplo, en las dificultades de aprendizaje del alumno.

De forma más pormenorizada se pueden señalar los siguientes extremos. La causa que recibe una mayor valoración media es la denominada: «la responsabilidad del alumno hacia el trabajo diario", con una valoración total media de 5,39; especialmente recibe una mayor por el profesorado (6,20 puntos) y es la causa más valorada por el alumnado. La variable «el compromiso del alumno con el estudio» es un ítem que recibe, también, altas puntuaciones $(6,36)$, es la puntuación más alta del profesorado $(6,29)$ y recibe una alta valoración tanto por el alumnado $(5,01)$ como por los equipos directivos $(5,91)$. Otra causa importante de fracaso escolar es "el apoyo familiar hacia el alumno" $(5,18)$. Como causas menos valoradas se encuentran: "los recursos económicos de la familia del alumno» $(4,14)$, «el cociente intelectual del alumno» $(4,16)$ y «la falta de habilidad del alumno para pedir ayuda a sus compañeros».

Si se estudian las escalas, se observa que la escala que más puntuación recibe es «la intervención del alumno y la familia en el aprendizaje» $(5,08)$. También es la escala más valorada por el conjunto de los informantes (4,78 para los alumnos; 5,80 para los profesores; y 5,64 para los equipos directivos). La siguiente escala es "la socialización del alumno en la escuela y la familia" (4,61). A las que le siguen la escala "La capacidad del alumno» $(4,50)$ y "La situación económica de la familia del alumno» $(4,16)$.

\subsection{Resultados de los análisis de correlación}

La Tabla 4 recoge las correlaciones de Pearson para las escalas del cuestionario administrado al alumnado.

TABLA 4

Correlaciones de Pearson para las escalas del cuestionario destinado al alumnado

\begin{tabular}{|l|c|c|c|c|}
\hline & INTERVENCIÓN & SOCIALIZACIÓN & CAPACITACIÓN & SITUACIÓN \\
\hline Intervención & 1 & & & \\
\hline Socialización & $0,450^{* *}$ & 1 & & \\
\hline Capacitación & $0,534^{* *}$ & $0,543^{* * *}$ & 1 & \\
\hline Situación & $0,377^{* *}$ & $0,231^{* * *}$ & $0,391^{* *}$ & 1 \\
\hline
\end{tabular}

NOTA: *** La correlación es significativa en el nivel 0,01 (2 colas). 
A. M. ANTELM, A. J. GIL, M. L. CACHEIRO Y E. PÉREZ NAVÍO CAUSAS DEL FRACASO ESCOLAR: UN ANÁLISIS DESDE LA PERSPECTIVA DEL PROFESORADO Y DEL ALUMNADO

Como señala la Tabla 4, las correlaciones más elevadas se encuentran en las escalas "Intervención" y "Capacitación" $(0,534)$ y "Capacitación" y "Socialización" $(0,543)$. Lo que vendría a señalar que las dificultades de aprendizaje del alumno se relacionan, en primer lugar, con sus habilidades de socialización en el aula y, en segundo lugar, con su esfuerzo diario.

La Tabla 5 recoge las correlaciones de Pearson para las escalas del cuestionario administrado al profesorado.

TABLA 5

Correlaciones de Pearson para las escalas del cuestionario destinado al profesorado

\begin{tabular}{|l|c|c|c|c|}
\hline & INTERVENCIÓN & SOCIALIZACIÓN & CAPACITACIÓN & SITUACIÓN \\
\hline Intervención & 1 & & & \\
\hline Socialización & $0,438^{* *}$ & 1 & & \\
\hline Capacitación & $0,420^{* *}$ & $0,633^{* *}$ & 1 & \\
\hline Situación & 0,125 & $0,342^{* *}$ & $0,346^{* *}$ & 1 \\
\hline
\end{tabular}

NotA: ** La correlación es significativa en el nivel 0,01 (2 colas).

Como muestra la Tabla 5, la escala "capacitación" se relaciona de forma más elevada con la escala «socialización». Lo que podría señalar que para el profesorado las dificultades del alumno se relacionan con un problema de socialización.

La Tabla 6 recoge las correlaciones de Pearson para las escalas del cuestionario administrado a los equipos directivos.

TABLA 6

Correlaciones de Pearson para las escalas del cuestionario destinado a los directivos

\begin{tabular}{|l|c|c|c|c|}
\hline & INTERVENCIÓN & SOCIALIZACIÓN & CAPACITACIÓN & SITUACIÓN \\
\hline Intervención & 1 & & & \\
\hline Socialización & $0,575^{* *}$ & 1 & & \\
\hline Capacitación & $0,392^{* * *}$ & $0,287^{* * *}$ & 1 & \\
\hline Situación & $0,330^{* *}$ & $0,264^{* *}$ & 0,162 & 1 \\
\hline
\end{tabular}

NOTA: *** La correlación es significativa en el nivel 0,01 (2 colas).

Como indica la Tabla 6, la correlación más elevada y significativa se observa entre las escalas "capacitación» e «intervención» (0,575). Para los equipos directivos las capacidades de los alumnos se relacionan con su trabajo diario. 

DEL PROFESORADO Y DEL ALUMNADO

\subsection{Resultados de los análisis de varianza}

En la Tabla 7 se presentan los resultados del primer análisis de varianza, ANOVA de un factor, para dar respuesta a la hipótesis 1. Como variables dependientes se toman cada una de las 16 causas de fracaso escolar y como variables independientes los 3 tipos de informantes (alumnado, profesorado y equipos directivos).

TABLA 7

ANOva de un factor con tres muestras:

la del alumnado, la del profesorado y la de los equipos

\begin{tabular}{|l|c|c|c|c|}
\hline \multicolumn{1}{|c|}{ ÍtEMS } & GL & $F$ & SIG. & $\begin{array}{c}\text { ETA } \\
\text { CUADRADO }\end{array}$ \\
\hline 1. El compromiso del alumnado con el estudio & 2 & 54,799 & 0,000 & 0,092 \\
\hline 2. Los recursos económicos de la familia & 2 & 11,329 & 0,000 & 0,020 \\
\hline 3. El apoyo familiar hacia el alumno & 2 & 62,089 & 0,000 & 0,102 \\
\hline 4. Las expectativas del alumno hacia el curso & 2 & 5,577 & 0,004 & 0,010 \\
\hline $\begin{array}{l}\text { 5. La responsabilidad del alumno hacia el } \\
\text { trabajo diario }\end{array}$ & 2 & 60,316 & 0,000 & 0,100 \\
\hline $\begin{array}{l}\text { 6. La percepción de autoeficacia o la propia } \\
\text { capacidad del alumno }\end{array}$ & 2 & 4,350 & 0,013 & 0,080 \\
\hline 7. La implicación de la familia en la educación & 2 & 114,360 & 0,000 & 0,174 \\
\hline 8. La falta de integración del alumno en el aula & 2 & 63,373 & 0,000 & 0,104 \\
\hline 9. El cociente intelectual del alumno & 2 & 23,330 & 0,000 & 0,041 \\
\hline 10. La situación laboral de la familia & 2 & 11,765 & 0,000 & 0,021 \\
\hline 11. Las dificultades de aprendizaje el alumno & 2 & 44,855 & 0,000 & 0,076 \\
\hline 12. La capacidad de autoevaluación del alumno & 2 & 32,128 & 0,000 & 0,056 \\
\hline $\begin{array}{l}\text { 13. La existencia de relaciones conflictivas en la } \\
\text { familia }\end{array}$ & 2 & 83,604 & 0,000 & 0,133 \\
\hline $\begin{array}{l}\text { 14. La falta de habilidad del alumno para pedir } \\
\text { ayuda o preguntar a los profesores }\end{array}$ & 2 & 49,201 & 0,000 & 0,083 \\
\hline 15. La resistencia a la frustración del alumno & 2 & 16,546 & 0,000 & 0,030 \\
\hline $\begin{array}{l}\text { 16. La falta de habilidad del alumno para pedir } \\
\text { ayuda a sus compañeros }\end{array}$ & 2 & 19,369 & 0,000 & 0,030 \\
\hline
\end{tabular}

NoTA: gl: grados de libertad

Como indica la Tabla 7, el conjunto de ítems son significativos ( sig. $\leq 0,05$ ) con excepción del ítem que corresponde a «la percepción de autoeficacia o la propia capacidad del alumno» (sig. 0,013). Por ello, se rechaza parcialmente la hipótesis 1 , 
A. M. ANTELM, A. J. GIL, M. L. CACHEIRO Y E. PÉREZ NAVÍO CAUSAS DEL FRACASO ESCOLAR: UN ANÁLISIS DESDE LA PERSPECTIVA DEL PROFESORADO Y DEL ALUMNADO

y existen diferencias significativas en la mayoría de las respuestas de los alumnos, los profesores y los equipos.

Por otro lado, la tabla muestra el tamaño del efecto a través de la «eta cuadrado». Así, el tamaño del efecto en la mayoría de los ítems es bajo (Cohen, 1988), se considera medio en los casos de la implicación de la familia en la educación $(0,174)$ y en la existencia de situaciones conflictivas en la familia $(0,133)$.

En la Tabla 8 se presentan los resultados del segundo análisis de varianza, ANOVA de un factor, para dar respuesta a la hipótesis 2. Como variables dependientes se presentan cada una de las 16 causas de fracaso escolar y como variables independientes se eligen los dos tipos de informantes, por un lado, el alumnado y, por otro, el conjunto de profesores y equipos.

TABLA 8

ANOva de un factor con dos grupos: la del alumnado y la del profesorado en su conjunto (profesores y directivos)

\begin{tabular}{|l|c|c|c|c|}
\hline \multicolumn{1}{|c|}{ ÍtEMS } & GL & $F$ & SIG. & $\begin{array}{c}\text { ETA } \\
\text { CUADRADO }\end{array}$ \\
\hline 1. El compromiso del alumnado con el estudio & 1 & 105,714 & 0,000 & 0,088 \\
\hline 2. Los recursos económicos de la familia & 1 & 17,461 & 0,000 & 0,016 \\
\hline 3. El apoyo familiar hacia el alumno & 1 & 123,911 & 0,000 & 0,102 \\
\hline 4. Las expectativas del alumno hacia el curso & 1 & 10,815 & 0,001 & 0,010 \\
\hline $\begin{array}{l}\text { 5. La responsabilidad del alumno hacia el } \\
\text { trabajo diario }\end{array}$ & 1 & 119,643 & 0,000 & 0,099 \\
\hline $\begin{array}{l}\text { 6. La percepción de autoeficacia o la propia } \\
\text { capacidad del alumno }\end{array}$ & 1 & 8,285 & 0,004 & 0,008 \\
\hline 7. La implicación de la familia en la educación & 1 & 228,572 & 0,000 & 0,173 \\
\hline 8. La falta de integración del alumno en el aula & 1 & 125,944 & 0,000 & 0,104 \\
\hline 9. El cociente intelectual del alumno & 1 & 46,177 & 0,000 & 0,041 \\
\hline 10. La situación laboral de la familia & 1 & 18,081 & 0,000 & 0,016 \\
\hline 11. Las dificultades de aprendizaje el alumno & 1 & 89,495 & 0,000 & 0,076 \\
\hline 12. La capacidad de autoevaluación del alumno & 1 & 64,299 & 0,000 & 0,056 \\
\hline $\begin{array}{l}\text { 13. La existencia de relaciones conflictivas en la } \\
\text { familia }\end{array}$ & 1 & 166,644 & 0,000 & 0,133 \\
\hline $\begin{array}{l}\text { 14. La falta de habilidad del alumno para pedir } \\
\text { ayuda o preguntar a los profesores }\end{array}$ & 1 & 96,812 & 0,000 & 0,082 \\
\hline 15. La resistencia a la frustración del alumno & 1 & 29,850 & 0,000 & 0,027 \\
\hline $\begin{array}{l}\text { 16. La falta de habilidad del alumno para pedir } \\
\text { ayuda a sus compañeros }\end{array}$ & 1 & 38,656 & 0,000 & 0.034 \\
\hline
\end{tabular}

NOTA: gl: grados de libertad 
A. M. ANTELM, A. J. GIL, M. L. CACHEIRO Y E. PÉREZ NAVÍO DEL PROFESORADO Y DEL ALUMNADO

Como se indica en la Tabla 8, el conjunto de ítems son significativos (sig. $\leq$ $0,05)$. Por ello, se rechaza la hipótesis 2 , por lo que existen diferencias significativas en las respuestas de los alumnos y del conjunto de profesores y equipos directivos. Además, se muestra el tamaño del efecto a través de la "eta cuadrado». El tamaño del efecto en la mayoría de los ítems es bajo (Cohen, 1988), se considera medio en los casos de la implicación de la familia en la educación $(0,173)$ y en la existencia de situaciones conflictivas en la familia $(0,133)$.

En la Tabla 9 se presentan los resultados del tercer análisis de varianza, ANOVA de un factor, para dar respuesta a la hipótesis 3. Como variables dependientes se presentan cada una de las 16 causas de fracaso escolar y como variables independientes se toman 2 tipos de informantes, por un lado el profesorado y, por otro, el equipo directivo.

\section{TABLA 9}

ANOva de un factor con dos grupos:

la del profesorado y la de los equipos directivos

\begin{tabular}{|l|c|c|c|c|}
\hline & GL & $F$ & SIG. & $\begin{array}{c}\text { ETA } \\
\text { CUADRADO }\end{array}$ \\
\hline 1. El compromiso del alumnado con el estudio & 1 & 8,321 & 0,004 & 0,024 \\
\hline 2. Los recursos económicos de la familia & 1 & 6,551 & 0,011 & 0,019 \\
\hline 3. El apoyo familiar hacia el alumno & 1 & 1,017 & 0,314 & 0,003 \\
\hline 4. Las expectativas del alumno hacia el curso & 1 & 0,582 & 0,446 & 0,002 \\
\hline $\begin{array}{l}\text { 5. La responsabilidad del alumno hacia el trabajo } \\
\text { diario }\end{array}$ & 1 & 2,565 & 0,110 & 0,008 \\
\hline $\begin{array}{l}\text { 6. La percepción de autoeficacia o la propia capacidad } \\
\text { del alumno }\end{array}$ & 1 & 4,495 & 0,482 & 0,001 \\
\hline 7. La implicación de la familia en la educación & 1 & 0,883 & 0,348 & 0,003 \\
\hline 8. La falta de integración del alumno en el aula & 1 & 1,608 & 0,206 & 0,005 \\
\hline 9. El cociente intelectual del alumno & 1 & 0,836 & 0,361 & 0,002 \\
\hline 10. La situación laboral de la familia & 1 & 7,640 & 0,006 & 0,022 \\
\hline 11. Las dificultades de aprendizaje el alumno & 1 & 0,481 & 0,488 & 0,001 \\
\hline 12. La capacidad de autoevaluación del alumno & 1 & 0,019 & 0,890 & 0,000 \\
\hline 13. La existencia de relaciones conflictivas en la familia & 1 & 1,657 & 0,199 & 0,005 \\
\hline $\begin{array}{l}\text { 14. La falta de habilidad del alumno para pedir ayuda } \\
\text { O preguntar a los profesores }\end{array}$ & 1 & 2,806 & 0,095 & 0,008 \\
\hline 15. La resistencia a la frustración del alumno & 1 & 4,332 & 0,038 & 0,013 \\
\hline $\begin{array}{l}\text { 16. La falta de habilidad del alumno para pedir ayuda } \\
\text { a sus compañeros }\end{array}$ & 1 & 0,187 & 0,665 & 0,001 \\
\hline
\end{tabular}

NOTA: gl: grados de libertad 
A. M. ANTELM, A. J. GIL, M. L. CACHEIRO Y E. PÉREZ NAVÍO CAUSAS DEL FRACASO ESCOLAR: UN ANÁLISIS DESDE LA PERSPECTIVA DEL PROFESORADO Y DEL ALUMNADO

Como se observa en la Tabla 9, la gran mayoría de ítems no son significativos (sig. $\geq 0,05$ ), con la excepción de "el compromiso del alumnado con el estudio" (sig. 0,000). Por ello, se acepta parcialmente la hipótesis 3, y mayoritariamente no existen diferencias significativas en las respuestas de los profesores y de los equipos directivos. También, la tabla muestra el tamaño del efecto a través de la «eta cuadrado". En este caso, el tamaño del efecto en la mayoría de los ítems es bajo (Cohen, 1988).

\section{DISCUSIÓN Y CONCLUSIONES}

Este trabajo ha tenido como objetivo principal analizar posibles causas relacionadas con el fracaso escolar. Como señala la literatura (Rumberger, 1987), el fracaso escolar se explica por la interacción entre factores individuales, características de comportamiento y rasgos ambientales, bajo esta perspectiva se ha abordado este trabajo.

Analizar la influencia del conjunto de causas es un tema clave de estudio en el problema del fracaso escolar, dicho de otra forma, analizar variables asociadas a este fenómeno es básico en la investigación del problema. Este análisis pasa, primero, por comprobar si las variables personales son más significativas que las sociales o si el sistema educativo puede dar claves en los procesos de intervención, los denominados programas de retención académica (Tinto, 1992). Segundo, por contrastar las relaciones entre los diversos grupos de variables, lo que permite conocer la influencia mutua de cada causa o grupos de causas en el problema del fracaso escolar (Fernández y Rodríguez, 2008). Este último extremo ha quedado de manifiesto con el análisis relacional que se ha realizado en este trabajo, pues se ha comprobado que, según la percepción del alumnado y del conjunto de profesorado, los problemas de socialización del alumno se relacionan con sus problemas de capacitación, por ejemplo, con sus dificultades de aprendizaje. Por lo que se podría asumir que estas causas se refuerzan y provocan mayores posibilidades de fracaso escolar.

El estudio en su conjunto aporta importantes conclusiones. La primera, conclusión relacionada con el primer objetivo, reconocer la percepción de causas de fracaso escolar por diferentes grupos de interés, se refiere a que existe cierta unanimidad en señalar y valorar las causas de fracaso escolar por los tres grupos de informantes: estudiantes, profesores y equipos directivos. En todos los casos, las causas de fracaso más valoradas se corresponden con la responsabilidad del alumno en el trabajo diario y la implicación del discente y su familia en el proceso de enseñanza y aprendizaje. Este tipo de causas también se ha destacado en otras investigaciones, Del Burgo (2002) señala que una de las principales causas de fracaso escolar es el bajo esfuerzo del alumnado y otros autores han señalado la importancia del compromiso del estudiante en el estudio del fracaso escolar (Archambault, Janosz, Fallu y Pagani, 2009). También son frecuentes los trabajos que achacan el fracaso escolar a la situación familiar o, más concretamente, a la 
A. M. ANTELM, A. J. GIL, M. L. CACHEIRO Y E. PÉREZ NAVÍO DEL PROFESORADO Y DEL ALUMNADO

falta de compromiso de la familia con la educación (Calero et al., 2010; Martín, Ariel, Marchena y Santana, 2015).

La segunda conclusión relacionada con el segundo objetivo, examinar la relación entre los grupos de causas de fracaso escolar por diferentes grupos de interés, hace referencia a que para el alumnado existen altas correlaciones entre, por un lado, capacitación y socialización y, por otro, capacitación e intervención. Lo que vendría a señalar que los problemas de aprendizaje del alumno se relacionan tanto con los problemas de intervención como con los problemas de integración en el aula y en la familia. Para el profesorado los problemas de aprendizaje se relacionan básicamente con la dificultad para la socialización. Y para los equipos directivos los problemas de aprendizaje se relacionan más fuertemente con la responsabilidad del alumno y de la familia en el aprendizaje. Estos resultados inciden en la importancia de la responsabilidad y de la adecuada socialización del alumno en sus problemas de aprendizaje (Fredricks, Blumenfeld y Paris, 2004).

La tercera conclusión, que está relacionada con el tercer objetivo, comparar la relación entre los grupos de causas de fracaso escolar por diferentes grupos de interés, se refiere a que, cuando se lleva a cabo un análisis de varianza para comprobar diferencias significativas entre los informantes, se puede observar dos diferentes visiones del fracaso escolar, la del alumnado y la del profesorado en su conjunto. Un ejemplo puede ilustrar estas diferencias. En el ítem referido a «la existencia de situaciones conflictivas en la familia" las valoraciones a esta cuestión son: 4,56 en el alumnado; 5,92 en el profesorado; y 6,07 en los directivos. Como se observa esta causa se valora más intensamente por el profesorado y en menor medida por el alumnado que puede vivir más directamente el problema. Estos resultados evidencian la importancia de profundizar en la visión de los alumnos acerca de las causas de fracaso.

Como conclusión final, se puede señalar que tanto en el presente estudio, como en el trabajo de Antelm et al. (2015), se destacan, como causas principales de fracaso escolar, la intervención del alumno y la familia en la enseñanza. Lo que vendría a señalar que la "responsabilidad", entendida como el valor que se da a la enseñanza, se considera una posible causa importante de fracaso escolar. A la vista de estos resultados, se podrían indicar algunas de las propuestas para involucrar a la familia en el centro escolar, por ejemplo: un contacto frecuente con la familia; mayor apoyo de los servicios sociales para ayudar a los déficits económicos y laborales; y una formación en competencias parentales que eviten el desapego (Álvarez y Martínez, 2016; Márquez, 2016; Serrano y Rodríguez, 2016; Méndez y Cerezo, 2018; Kattan y Székely, 2017).

Por otro lado, la organización escolar no es ajena a este problema, pues la prevención del fracaso escolar requiere una escuela adaptada a la realidad social de los alumnos, a través de un currículo actualizado con programas de estudio que respondan a sus necesidades e intereses (Ottosen, Goll y Sørlie, 2016; Martínez y Molina, 2017). La flexibilidad curricular y la atención individualizada son aspectos metodológicos que favorecen la inserción sociolaboral y la autoestima (Álvarez y 
A. M. ANTELM, A. J. GIL, M. L. CACHEIRO Y E. PÉREZ NAVÍO

CAUSAS DEL FRACASO ESCOLAR: UN ANÁLISIS DESDE LA PERSPECTIVA DEL PROFESORADO Y DEL ALUMNADO

Martínez, 2016; Domingo y Martos, 2017; Vázquez y Barrera, 2017; Ritacco y Amores, 2016), promoviendo la habilidad y esfuerzo, con el objetivo de aumentar su rendimiento académico y mejorar el ajuste social (Inglés, Aparisi, Delgado, Granados y García, 2017).

Se pueden mencionar algunas limitaciones de este trabajo. La primera limitación se refiere a que la investigación se basa en la percepción de fracaso escolar de los tres grupos de informantes, no se comprueba con casos reales de fracaso escolar. En posteriores investigaciones se trataría de analizar si la causa principal de fracaso escolar se relaciona con la percepción de los sujetos. Un estudio de casos ayudaría en este sentido. La segunda limitación se refiere a que se trata de un estudio descriptivo, no se plantean relaciones de causalidad. En posteriores investigaciones se puede abordar este tipo de estudios, que permitiría analizar más en profundidad las causas que pudieran tener una mayor influencia en los casos de fracaso escolar. Además, se podría investigar a otros interesados en el tema de fracaso escolar, como los padres o tutores, para comparar las diferentes respuestas y sus percepciones sobre el tema de fracaso. Todo ello podría facilitar el conocimiento de estrategias de intervención tendentes a paliar al problema del fracaso escolar (Vega y Aramendi, 2009).

Se trataría, en la línea de trabajos como los de Lessard, Butler, Lortin y Marcotte (2014), de llevar a cabo estudios cualitativos desde una perspectiva a largo plazo en la que los adultos puedan ayudar y orientar a estos estudiantes a identificar sus intereses, valores y aptitudes (González-Flores, Sevillano y Rey, 2007). Se trata de llevar a cabo acciones de prevención, pues, como señalan Miñaca y Hervás (2013), aunque el estudio del fracaso escolar se centra en la educación secundaria, existen indicios en educación primaria con alumnos que no finalizan y van acumulando retraso y faltas de asistencia a clase no justificadas. Estas autoras realizan una revisión de los programas de intervención dirigidos a la prevención del fracaso y abandono escolar y seleccionan ocho en los que han propiciado resultados positivos: aumento de la sensibilidad de los sectores de la comunidad y las familias; disminución de la tasa de absentismo; reducción de conductas disruptivas; mejora de la autoestima y control de las emociones; y mejora en el éxito escolar, entre otros.

\section{REFERENCIAS BIBLIOGRÁFICAS}

Aguado, G. O.; Aguilar, M. A. y González, N. N. (2009). El impacto de las representaciones sociales de los actores educativos en el fracaso escolar. Revista Iberoamericana de Educación, 51, 23-32.

Álvarez, L. y Martínez, R. A. (2016). Cooperación entre las familias y los centros escolares como medida preventiva del fracaso y del riesgo de abandono escolar en adolescentes. Revista Latinoamericana de Educación Inclusiva, 10 (1), 175-192.

Antelm, A. M.; Gil, A. J. y Cacheiro, M. L. (2015). Análisis de las causas del fracaso escolar desde la perspectiva del alumnado del último curso de Educación Secundaria Obligatoria en España y su relación con el estilo de aprendizaje. Educación y Educadores, 18 (3), 471-489. 
A. M. ANTELM, A. J. GIL, M. L. CACHEIRO Y E. PÉREZ NAVÍO

CAUSAS DEL FRACASO ESCOLAR: UN ANÁLISIS DESDE LA PERSPECTIVA DEL PROFESORADO Y DEL ALUMNADO

Archambault, I.; Janosz, M.; Fallu, J. F. y Pagani, L. S. (2009). Student engagement and its relationship with early high school dropout. Journal of Adolescence, 32, 651-670.

Bratti, M. (2007). Parents' income and children's school drop-out at 16 in England and wales: Evidence from the 1970 British cohort study. Review of Economic Household, 5, 515540.

Britner, S. L. y Pajares, F. (2006). Sources of science self-efficacy beliefs of middle school students. Journal of Research in Science Teaching, 43 (5), 485-499.

Cabo, A. y Romero, C. (2014). Intervención socioeducativa interdisciplinar con jóvenes en riesgo de exclusión social: el centro socioeducativo Díaz-Pintado de Burjassot. EDETANIA, 45, 219-234.

Calero, J.; Choi, A. y Waisgrais, S. (2010). Determinantes del riesgo de fracaso escolar en España: una aproximación a través de un análisis logístico multinivel aplicado a PISA2006. Revista de Educación, número extraordinario, 225-256.

Castejón, J. L. y Vera, M. I. (1996). A causal model about the individual and contextual determinants of academic achievement. The High School Journal, 80 (1), 20-29.

Cohen, J. (1988). Statistical power analysis for the behavioral sciences (2. ${ }^{\text {nd }}$ Ed.). Hillsdale, NJ: Lawrence Earlbaum Associates.

De Witte, K.; Cabus, S.; Thyssen, G.; Groot, W. y Brink, H. M. (2014). A Critical Review of the Literature on School Dropout. TIER Working Paper Series, 14/14.

Del Burgo, M. (2002). El fracaso escolar. Madrid: Acento.

Domingo, J. y Martos, J. M. (2017). Prevención del Fracaso Escolar en Educación Secundaria en Andalucía desde la Voz del Profesorado. Estudio de Caso. Revista Internacional de Educación para la Justicia Social, 6 (1), 329-347.

Engin-Demir, C. (2009). Factors Influencing the Academic Achievement of the Turkish Urban Poor. International Journal of Educational Development, 29, 17-29.

Escudero, J. M. y Martínez, B. (2012). Las políticas de lucha contra el fracaso escolar: ¿programas especiales o cambios profundos del sistema y la educación? Revista de Educación, número extraordinario, 174-193.

Espínola, V. y Claro, J. P. (2010). Estrategias de prevención de la deserción en la Educación Secundaria: perspectiva latinoamericana. Revista de Educación, número extraordinario, 257-280.

Fernández, J. J. y Rodríguez, J. R. (2008). Los orígenes del fracaso escolar en España. Un estudio empírico. En V. Pérez-Díaz (Coord.). Mediterráneo Económico (pp. 323-350). Málaga: CAJAMAR Caja Rural, Sociedad Cooperativa.

Fredricks, J. A.; Blumenfeld, P. C. y Paris, A. H. (2004). School engagement: potential of the concept, state of the evidence. Review of Educational Research, 74 (1), 59-109.

González, C.; Caso, J.; Díaz, K. y López, M. (2012). Rendimiento académico y factores asociados. Aportaciones de algunas evaluaciones a gran escala. Bordón, Revista de Pedagogía, 64 (2), 51-68.

González, M. T. (2006). Absentismo y abandono escolar: Una situación singular de la exclusión educativa. REICE, Revista Electrónica Iberoamericana sobre Calidad, Eficacia y Cambio en Educación, 4 (1), 1-15.

González-Flores, M. P.; Sevillano, M. L. y Rey, L. (2007). Promover en los adolescentes la identificación de sus valores como factores que les protegen de las adicciones: un modelo vivencial centrado en el estudiante. Enseñanza \& Teaching, 25, 157-178.

Hafiane, S.; Kaddari, F.; Elachqar, A.; Elaissaoui, K. y Tahraoui, A. (2015). Major factors that influence school failure in the Northern Region of Morocco (Fez-Boulemane as a case study). Procedia - Social and Behavioral Sciences, 197, 2164-2173. 
A. M. ANTELM, A. J. GIL, M. L. CACHEIRO Y E. PÉREZ NAVÍO CAUSAS DEL FRACASO ESCOLAR: UN ANÁLISIS DESDE LA PERSPECTIVA DEL PROFESORADO Y DEL ALUMNADO

Inglés, C. J.; Aparisi, D.; Delgado, B.; Granados, L. y García, J. M. (2017). Relationship between sociometrist type and self-attributions for academic failure in a Spanish sample from secondary education. Electronic Journal of Research in Educational Psychology, 15 (2), 398-421.

Kattan, R. B. y Székely, M. (2017). Analyzing Upper Secondary Education Dropout in Latin America through a Cohort Approach. Journal of Education and Learning, 6 (4), 12-39.

Lessard, A.; Butler-Kisber, L.; Fortin, L. y Marcotte, D. (2014). Analyzing the Discourse of Dropouts and Resilient Students. Journal of Educational Research, 107 (2), 103-110.

Levin, B. (2003). Approaches to equity in policies for lifelong learning. Equity in education series. Paris: OECD. Recuperado de http://www.oecd.org/education/school/38692676. pdf.

Lozano, A. (2012). Personal, family, and academic factors affecting low achievement in secondary school. Electronic Journal of Research in Educational Psychology and Psychopedagogy, 1 (1), 43-66.

Marchesi, A. (2003). El fracaso escolar en España. Madrid: Fundación Alternativas. Documento de Trabajo 11/2003. Recuperado de https://www.nodo50.org/movicaliedu/ fracasoescolarespana.pdf.

Márquez, C. (2016). Factores asociados al fracaso escolar en la educación secundaria de Huelva. REICE. Revista Iberoamericana Sobre Calidad, Eficacia y Cambio en Educación, 14 (2), 131-144.

Martín, J. C.; Ariel, J.; Marchena, R. y Santana, R. (2015). Educación parental y competencias parentales para prevenir el abandono escolar temprano. Bordón, Revista de Pedagogía, 67 (4), 73-92.

Martínez, E. y Molina, E. (2017). Incidencia de factores académicos en el fracaso escolar. Reflexiones derivadas de la experiencia de profesores jubilados. Profesorado, Revista de Currículum y Formación del Profesorado, 21 (1), 191-211.

Martínez-Otero, V. M. (2009). Investigación y reflexión sobre condicionantes del fracaso escolar. Revista Latinoamericana de Estudios Educativos, 39 (1), 11-38.

Melendro, M. (2008). Absentismo y fracaso escolar: la educación social como alternativa. Bordón, Revista de Pedagogía, 60 (4), 65-77.

Mena, L.; Fernández, M. y Riviére, J. (2010). Desenganchados de la educación: procesos, experiencias, motivaciones y estrategias del abandono y del fracaso escolar. Revista de Educación, número extraordinario, 119-145.

Méndez, I. y Cerezo, F. (2018). La repetición escolar en educación secundaria y factores de riesgo asociados. Educación XXI, 21 (1), 41-62.

Migali, G. y Zucchelli, E. (2017). Personality traits, forgone health care and high school dropout: Evidence from us adolescents. Journal of Economic Psychology, 62, 98-119.

Miñaca, M. I. y Hervás, M. (2013). Intervenciones dirigidas a la prevención del fracaso y abandono escolar: un estudio de revisión. Revista Española de Educación Comparada, 21, 203-220.

Nunnally, J. y Bernstein, I. (1994). Psychometric theory. New York: McGraw-Hill.

Oreopoulos, P. (2007). Do dropouts drop out too soon? Wealth, health and happiness from compulsory schooling. Journal of Public Economics, 91, 2213-2229.

Ottosen, K. O.; Goll, C. B. y Sørlie, T. (2016). The multifaceted challenges in teacher-student relationships: A qualitative study of teachers' and principals' experiences and views regarding the dropout rate in Norwegian upper-secondary education. Scandinavian Journal of Educational Research, 61 (3), 354-368. 
A. M. ANTELM, A. J. GIL, M. L. CACHEIRO Y E. PÉREZ NAVÍO

CAUSAS DEL FRACASO ESCOLAR: UN ANÁLISIS DESDE LA PERSPECTIVA DEL PROFESORADO Y DEL ALUMNADO

Pérez, G. (2008). La educación como respuesta a los retos que plantea la escuela. Bordón, Revista de Pedagogía, 60 (4), 15-29.

Perrenoud, P. (1990). La construcción del éxito y fracaso escolar: hacia un análisis del éxito, del fracaso y de las desigualdades como realidades construidas por el sistema escolar. Madrid: Morata.

Rindskopf, D. (2015). Reliability: measurement. En J. D. Wright (Ed.). International encyclopedia of the social \& behavioral sciences (pp. 248-252). Oxford: Elsevier.

Ritacco, M. y Amores, F. J. (2016). Estudiantes en riesgo de exclusión educativa en secundaria. Percepciones del profesorado implicado en programas extraordinarios. Enseñanza E Teaching, 34 (1), 137-160.

Robison, S.; Jaggers, J.; Rhodes, J.; Blackmon, B. R. y Church, W. (2017). Correlates of educational success: Predictors of school dropout and graduation for urban students in the Deep South. Children and Youth Services Review, 73, 37-46.

Roca, E. (2010). El abandono temprano de la educación y la formación en España. Revista de Educación, número extraordinario, 31-62.

Rumberger, R. W. (2012). Activity matters: Understanding student interest in school science. Journal of Research in Science Teaching, 57 (4), 101-122.

Serrano, B. y Rodríguez, M. P. (2016). Funcionalidad de la familia y su incidencia en el rendimiento académico en adolescentes. Revista Didascalia: Didáctica y Educación, 7 (1), 235-255.

Swarat, S.; Ortony, A. y Revelle, W. (2012). Activity matters: Understanding student interest in school science. Journal of Research in Science Teaching, 49 (4), 515-537.

Tinto, V. (1992). El abandono de los estudios superiores: una nueva perspectiva de las causas del abandono y su tratamiento. México: Universidad Nacional Autónoma de México.

Vázquez, M. y Barrera, E. (2017). Metodología para la inserción social de jóvenes con fracaso escolar. Nuevos escenarios metodológicos especializados. IJERI, International Journal of Educational Research and Innovation, 9, 105-121.

Vega, A. y Aramendi, P. (2009). La atención a la diversidad: interrogantes para la iniciación profesional de los fracasados. Enseñanza \& Teaching, 27, 157-170.

Willms, D. J. y Somers, M. A. (2001). Family, Classroom, and School effects on Children's Educational Outcomes in Latin America. School Effectiveness and School Improvement, 12 (4), 409-445. 